\title{
Electrochemical and analytical performance of boron-doped diamond electrode for determination of ascorbic acid
}

\author{
Ivana Sálusováa, Kristína Cinkováa, Barbora Brtkováa, \\ Marian Vojs ${ }^{b}$, Marián Marton ${ }^{b}$, Lubomír Švorc ${ }^{a}$ \\ ${ }^{a}$ Institute of Analytical Chemistry, Faculty of Chemical and Food Technology, \\ Slovak University of Technology in Bratislava, Radlinského 9, SK-812 37 Bratislava, Slovak Republic \\ ${ }^{b}$ Institute of Electronics and Photonics, Faculty of Electrical Engineering and Information Technology, \\ Slovak University of Technology in Bratislava, Ilkovičova 3, SK-812 19 Bratislava, Slovak Republic \\ lubomir.svorc@stuba.sk
}

\begin{abstract}
The electrochemical behavior and determination of ascorbic acid (AA) was investigated at a bare boron-doped diamond (BDD) electrode using cyclic and differential pulse voltammetry. The influence of $\mathrm{pH}$ of supporting electrolyte and scan rate on the current response of analyte was examined to select the suitable experimental conditions. It was found that AA provided one well-shaped irreversible and diffusioncontrolled oxidation peak at $+0.87 \mathrm{~V}$ ws. $\mathrm{Ag} / \mathrm{AgCl}$ in Britton-Robinson buffer $\mathrm{pH}$ 4.0. Applying differential pulse voltammetry, the peak current of AA was linearly proportional to its concentration from $5 \times 10^{-6}$ to $2 \times 10^{-4} \mathrm{~mol} \mathrm{~L}^{-1}\left(R^{2}=0.999\right)$, with the limit of detection of $1.1 \times 10^{-6} \mathrm{~mol} \mathrm{~L}^{-1}$ and the good repeatability (relative standard deviation of $2.3 \%$ ). The developed electroanalytical protocol was successfully applied to determine the content of AA in commercial pharmaceutical preparations, based on the standard additions method, with the obtained recovery of $122 \%$. The accomplished analytical performance indicates that BDD electrodes are promising electrochemical sensors for pharmaceutical analysis.
\end{abstract}

Keywords: ascorbic acid, boron-doped diamond, differential pulse voltammetry, limit of detection, pharmaceutical analysis

\section{Introduction}

Ascorbic acid (AA) is the vitamin consumed worldwide on a large scale as an antioxidant agent in food, beverages and medicine. It is present in many biological systems and in multivitamin preparations, which are widely used for the prevention and treatment of common cold, mental illness and infertility (Lee et al., 2016). Besides, AA is an important agent in several enzymatic reactions and in cell defense against oxidative stress which may lead to disruption of a living cell or to molecular and cellular DNA damage (Mason et al., 2016). Hence, for the potential role of AA in preventing diseases, great attention has been devoted to this vitamin along several decades. The current challenge of chemists is to develop novel analytical methods and protocols for its simple and rapid detection and quantification.

Different analytical methods have been employed to determine $\mathrm{AA}$ in pharmaceuticals, food and biological fluids, mostly including separation methods such as high-performance liquid chromatography (Zuo et al., 2015) and capillary zone electrophoresis (Falkova et al., 2015). These techniques are accurate, precise and robust, but they are not as expeditious as required for routine control analysis. Several spectral methods have also been employed, most of them based on UV (Bi et al., 2016), fluorescence (Fong et al., 2016) and Raman spectroscopy (Neuberger et al., 2017), however they are time-consuming and not easily applied to colored or turbid samples. As AA is the electroactive substance, electrochemical methods are one of the most favorable techniques for its determination. Moreover, these methods overcome the shortcomings of separation and spectral methods because of low cost, suitability of miniaturization and easy operation while providing comparable sensitivity. The electrochemical behavior and determination of AA have been extensively studied along last decades using various conventional materials based on carbon paste electrode (CPE) (Pisoschi et al., 2011), glassy carbon electrode (GCE) (Guanghan et al., 1994), gold electrode (Muñoz et al., 2001) and carbon paste electrode (CPE) (Pisoschi et al., 2011). However, a major obstacle in the detection of this vitamin in biological samples are interferences from other electroactive constituents, such as dopamine and uric acid, which undergo the oxidation at almost the same potential as AA at conventional electrodes. Therefore, various chemically modified electrodes (CMEs) have been used in order to improve current response of AA, thus giving rise to higher sensitivity and allowing potential separation of their oxidation peaks (Ensafi et al., 2010). 
Recently, CMEs with an excellent electrocatalytic activity and fast response time towards the oxidation of AA using GCE modified by cobalt (II) phthalocyanine-multi-walled carbon nanotubes (Zuo et al., 2012), poly(l-leucine)/DNA (Zheng et al., 2015), octacyanomolybdate-doped-poly(4-vinylpyridine) (Thangamuthu et al., 2007), poly-xanthurenic acid (poly-Xa)/multi-walled carbon nanotubes (Silva et al., 2012), ionic liquid-carbon nanotubes (Ping et al., 2012), carbon-supported PdNi nanoparticles (Zhang et al., 2013), cobalt film (Tian et al., 2010) and single-walled carbon nanotubes (Habibi et al., 2011) have been reported.

Concerning bare and non-traditional electrode materials, a boron-doped diamond (BDD) is considered as one of the most promising platform to be a working electrode for individual and simultaneous determination of various biologically active substances (Svítková et al., 2016). The choice of this material in this work was based on its interesting properties, compared with conventional ones (graphite, carbon paste, glassy carbon, gold), such as stability in both aqueous and non-aqueous electrolytes, negligible adsorption of organic molecules, low and stable background current as well as low capacitance, resulting in a low limit of detection (Cinková et al., 2016). Moreover, differential pulse voltammetry coupled to the unique properties of BDD electrode could offer the possibility of determining the concentration of analyte directly in the sample without any pretreatment or chemical separation. These characteristics make $\mathrm{BDD}$ electrodes very competitive in electroanalytical chemistry, oftentimes providing one of the best choice for determination of electroactive compounds and being suitable also for routine analysis.

In this paper, the electrochemical and analytical performance of the highly doped BDD electrode for the AA determination was explored using cyclic and differential pulse voltammetry. The developed electroanalytical protocol was applied to the analysis of commercial pharmaceuticals, thus representing the low cost, simple and rapid alternative to separation and spectral methods.

\section{Materials and Methods}

Cyclic voltammetric (CV) and differential pulse voltammetric (DPV) experiments were performed using Autolab PGSTAT 101 (Metrohm Autolab B.V., The Netherlands), controlled by the NOVA (version 1.8) software. The electrochemical cell was arranged with a highly doped BDD working electrode (inner diameter of BDD film of $740 \mu \mathrm{m}, \mathrm{B} / \mathrm{C}$ ratio in gaseous phase of $8000 \mathrm{ppm}$ ), an $\mathrm{Ag} / \mathrm{AgCl} / 3 \mathrm{~mol}$ $\mathrm{L}^{-1} \mathrm{KCl}$ reference electrode and a $\mathrm{Pt}$ wire as a counter electrode. All $\mathrm{pH}$ values were assessed using $\mathrm{pH}$ enomenal ${ }^{\circledR} \mathrm{pH} 1100 \mathrm{~L}$ meter (VWR, Slovakia) with a combined electrode (glass-reference electrode).

Ascorbic acid (AA, purity of $99 \%$ ) was purchased from Sigma Aldrich (Slovakia) and used without any further purification. The stock solution of AA $\left(1.0 \times 10^{-3} \mathrm{~mol} \mathrm{~L}^{-1}\right)$ was prepared by dissolution of the particular amount in deionized water. The working solutions of lower concentrations were made of the stock solution by suitable dilution with particular supporting electrolyte directly in electrochemical cell. Britton-Robinson (B-R) buffer was prepared by mixing of $0.04 \mathrm{~mol} \mathrm{~L}^{-1}$ of phosphoric acid, acetic acid and boric acid and adjusting with sodium hydroxide $\left(0.2 \mathrm{~mol} \mathrm{~L}^{-1}\right)$ to desired $\mathrm{pH}$ value. All chemicals were of analytical grade purity. The deionized water with resistivity higher than $18 \mathrm{M} \Omega \mathrm{cm}$ was used throughout the voltammetric measurements.

Prior to initiating the electrochemical measurements, the BDD electrode surface was always washed down with deionized water and then pretreated by simple cycling in $1.5 \mathrm{~mol} \mathrm{~L}^{-1}$ sulphuric acid using potentials in the range from -2.0 to $+2.0 \mathrm{~V}$ until a stable signal was observed (usually 5 cycles in $\mathrm{CV}$ ). The DPV instrumental parameters were set as follows: modulation amplitude of $50 \mathrm{mV}$, modulation time of $50 \mathrm{~ms}$ and scan rate of $10 \mathrm{mV} \mathrm{s}^{-1}$. The linear least-squares regression (OriginPro 8.5, OriginLab Corporation, USA) was used for the evaluation of the calibration curve with the relevant results (slope and intercept) with a confidence interval for $95 \%$ probability. The limit of detection (LOD) and limit of quantification (LOQ) was calculated as three and ten times the standard deviation of the intercept divided by the slope of calibration curve, respectively.

Commercial pharmaceutical tablets Celaskon ${ }^{\circledR}$ 250 (Zentiva, Czech Republic) with the declared content of AA $(250 \mathrm{mg})$ were bought in a local pharmacy. The sample stock solution was prepared according to the following procedure: ten tablets were accurately weighed (mean weight of one tablet was found to be $342 \mathrm{mg}$ ) and powdered by pestle in mortar. An equivalent portion of $346 \mathrm{mg}$ of homogenized powder was dissolved in deionized water. The mixture was subsequently filtered, then transferred into $50 \mathrm{~mL}$ volumetric flask and finally filled up to the mark with deionized water to get stock sample solution. $20 \mu \mathrm{L}$ of stock sample solution was added to the electrochemical cell to $20 \mathrm{~mL}$ of the supporting electrolyte. The standard addition method with corresponding volumes of 0.7 , 1.4 and $2.1 \mathrm{~mL}$ of $1.0 \times 10^{-3} \mathrm{~mol} \mathrm{~L}^{-1} \mathrm{AA}$ standard solution was used to quantify the vitamin content in pharmaceuticals. 


\section{Results and Discussion}

A study of $\mathrm{pH}$ effect of the supporting electrolyte on current response is an important part within a characterization of electrochemical behavior of an analyte at the particular working electrode. From electrochemical viewpoint, AA is an analyte known for its irreversible oxidation at different (bare and modified) electrode surfaces under various experimental conditions (Pisoschi et al., 2011; Zheng et al., 2015). Initially, the $\mathrm{pH}$ effect on the DPV response of $1.0 \times 10^{-4} \mathrm{~mol} \mathrm{~L}^{-1} \mathrm{AA}$ was examined at $\mathrm{pHs}$ of 2-12 using B-R buffers in the potential range from +0.2 to $+1.2 \mathrm{~V}$ (Fig. 1). It was found that the redox reaction of AA at $\mathrm{BDD}$ electrode was manifested by the presence of distinct oxidation peak which was found to be $\mathrm{pH}$-dependent. The best results were attained in mildly acidic environment ( $\mathrm{pH} 2.0-5.0)$. The peak current of AA $\left(I_{\mathrm{p}}\right)$ was increasing from pH 2.0 to 4.0, then decrease slightly for $\mathrm{pH} 5.0$ and subsequently a significant decline was noticed when $\mathrm{B}-\mathrm{R}$ buffer in the range of $\mathrm{pH} 6.0-12.0$ was used, as demonstrated in Fig. 2. Furthermore, it is evident that the $\mathrm{pH}$ increase produced a negative shift in the peak potential $\left(E_{\mathrm{p}}\right)$ which confirmed the participation of protons in the electrode reaction of $\mathrm{AA}$ at $\mathrm{BDD}$ electrode. However, no reasonable slope value of $E_{\mathrm{p}}$ vs. $\mathrm{pH}$ depedence furnishing the information about the number of exchanged protons and electrons was ascertained. Overall, B-R buffer $\mathrm{pH} 4.0$ was used for further voltammetric studies as it provided the highest peak current for the oxidation of AA.

Cyclic voltammetry (CV) was used to investigate the electrochemical behaviour of $\mathrm{AA}$ at bare $\mathrm{BDD}$ electrode, recorded in the absence (blank measurement) and in the presence of $1.0 \times 10^{-4} \mathrm{~mol}$ $\mathrm{L}^{-1} \mathrm{AA}$ in the potential range from -0.5 to $+1.4 \mathrm{~V}$ at a scan rate of $100 \mathrm{mV} \mathrm{s}^{-1}$ in $\mathrm{B}-\mathrm{R}$ buffer $\mathrm{pH} 4.0$. As can be inferred from the $\mathrm{CV}$ records shown in Fig. 3, an oxidation peak of AA at $+0.87 \mathrm{~V}$ was observed at forward scan with the absence of any reduction peak at reverse scan, obviously confirming the totally irreversible electrode reaction of vitamin at $\mathrm{BDD}$ electrode. This behavior is mostly consistent with those reported for CPE (Pisoschi et al., 2011), GGE (Guanghan et al., 1994) and CMEs (Silva et al., 2012). Besides, it is also apparent from Fig. 3 that the background current of the BDD electrode was found to be relatively low at the higher positive potentials, thus approving excellent properties of this material for electroanalytical purposes. Taken into consideration the studies published in literature so far, the electrochemical oxidation of AA occurs with the elimination of two protons and two electrons to form dehydroascorbic acid.

To assess the adsorption or the diffusion as the rate-determining step of the AA oxidation mechanism at the BDD electrode, the influence of the scan rate $(v)$ was investigated by $\mathrm{CV}$ in the range from 0.005 to $0.5 \mathrm{~V} \mathrm{~s}^{-1}$. Due to the fact that the hydrogen terminated BDD electrode used in this work should exhibit low adsorption properties (Cinková et al., 2016), a diffusion-controlled redox reaction of AA was expected to be observed for this advanced electrode material. As depicted in Fig. 4, the oxidation peaks of $1.0 \times 10^{-4} \mathrm{~mol} \mathrm{~L}^{-1} \mathrm{AA}$ in $\mathrm{B}-\mathrm{R}$ buffer $\mathrm{pH} 4$ were found to be linearly dependent

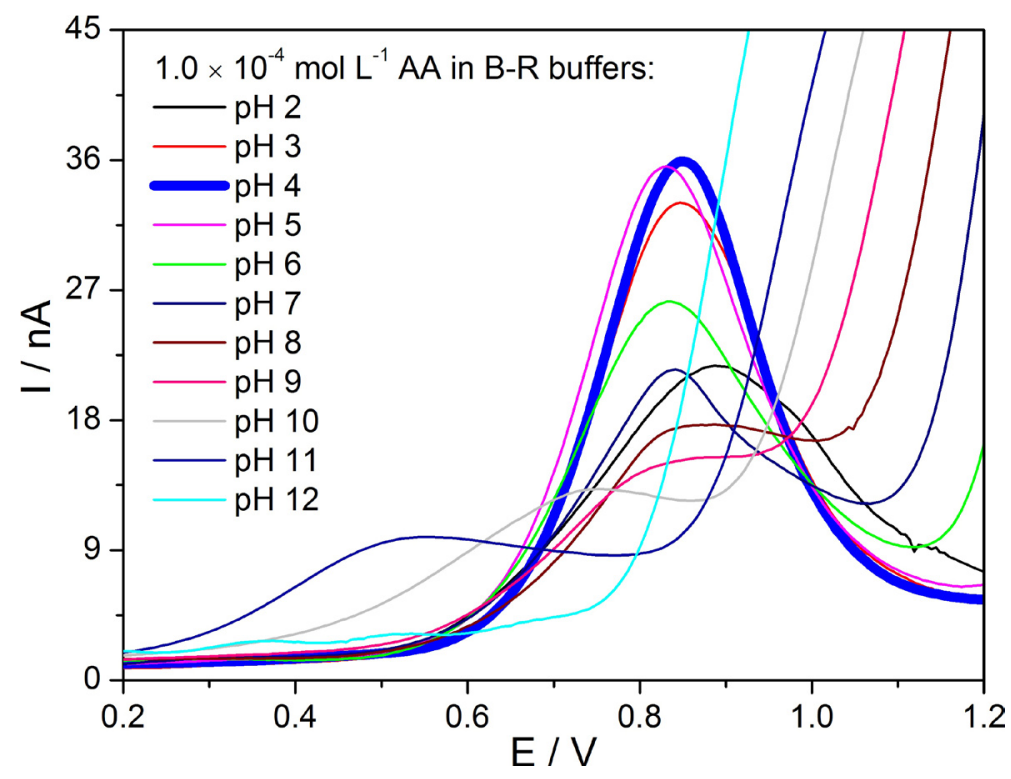

Fig. 1. DPV records of $1.0 \times 10^{-4} \mathrm{~mol} \mathrm{~L}^{-1} \mathrm{AA}$ in B-R buffers $(\mathrm{pH} 2.0-12.0)$ at BDD electrode. DPV parameters: modulation amplitude of $50 \mathrm{mV}$, modulation time of $50 \mathrm{~ms}$ and scan rate of $10 \mathrm{mV} \mathrm{s}^{-1}$. 


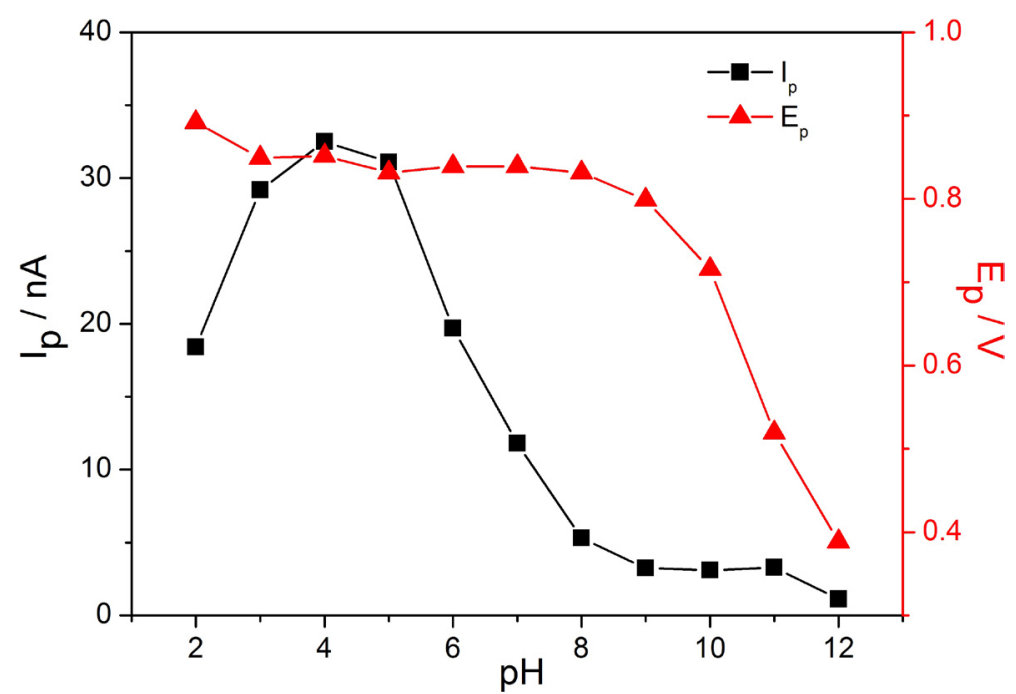

Fig. 2. Effect of $\mathrm{pH}$ of $\mathrm{B}-\mathrm{R}$ buffers on the peak current $\left(I_{\mathrm{p}}\right)$ and the peak potential $\left(E_{\mathrm{p}}\right)$ of $1.0 \times 10^{-4} \mathrm{~mol} \mathrm{~L}^{-1} \mathrm{AA}$ using DPV at BDD electrode. DPV parameters: modulation amplitude of $50 \mathrm{mV}$, modulation time of $50 \mathrm{~ms}$ and scan rate of $10 \mathrm{mV} \mathrm{s}^{-1}$.

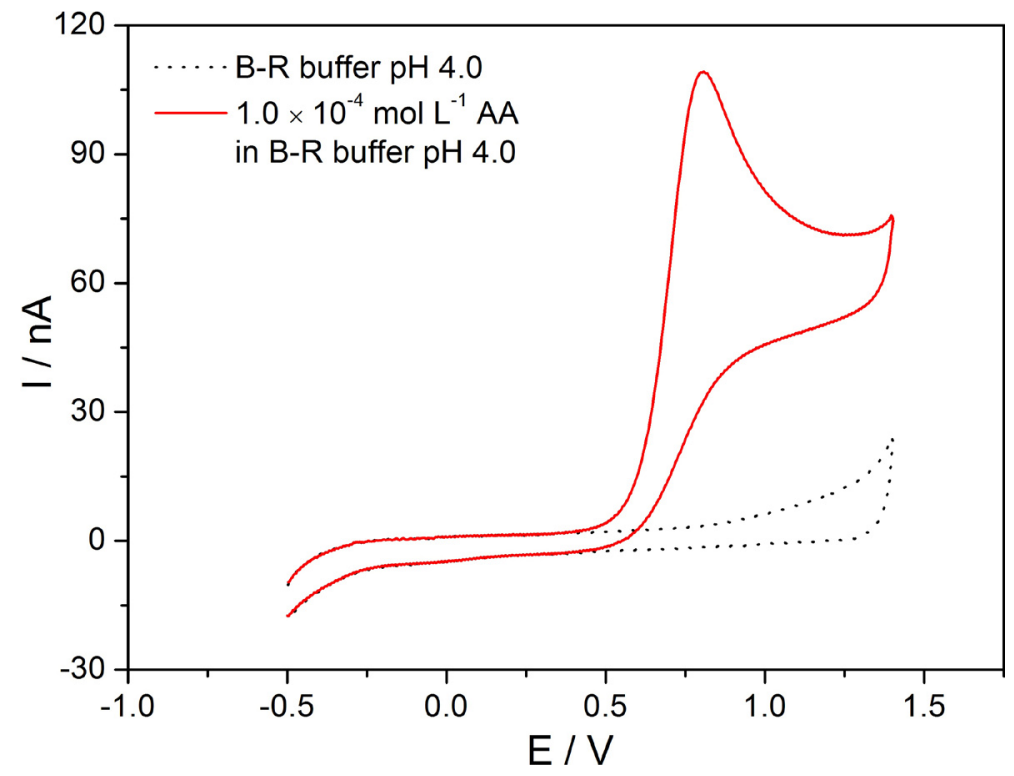

Fig. 3. CV records of supporting electrolyte (B-R buffer $\mathrm{pH} 4.0$ ) and $1.0 \times 10^{-4} \mathrm{~mol} \mathrm{~L}^{-1} \mathrm{AA}$ in B-R buffer $\mathrm{pH} 4.0$ at BDD electrode with a scan rate of $100 \mathrm{mV} \mathrm{s}^{-1}$.

on $v^{1 / 2}$ values with a slight shift of peak potentials to more positive values. The corresponding regression equation (Eq. 1) may be expressed:

$$
\begin{gathered}
I_{\mathrm{p}}(\mathrm{nA})=(17 \pm 4)+(126 \pm 9) \times v^{1 / 2}\left(\mathrm{~V} \mathrm{~s}^{-1}\right), \\
R^{2}=0.954
\end{gathered}
$$

The linear correlation indicated the fact that the redox process of AA was controlled by diffusion rather than adsorption at the BDD electrode and the observed phenomenon is in agreement with the electrochemical behavior of structurally and biologically related compounds investigated at this advanced electrode material (Švorc et al., 2015). In addition, plot of the $\log I_{\mathrm{p}}$ vs. $\log v$ dependence (inset of Fig. 4, Eq. 2) was appeared to be linear, with the slope value of 0.37 , in close conformity with the theoretical value $(0.50)$ for a diffusion-controlled process (Compton et al., 2014).

$$
\begin{gathered}
\log I_{\mathrm{p}}=(-6.86 \pm 0.02)+(0.37 \pm 0.01) \times \log v, \\
R^{2}=0.988
\end{gathered}
$$

The analytical performance of the BDD electrode was investigated by detecting different AA concentrations using DPV in B-R buffer $\mathrm{pH}$ 4.0. The voltammetric profile showing distinct oxidation peaks of $\mathrm{AA}$ at $+0.7 \mathrm{~V}$ and the linear correlation 


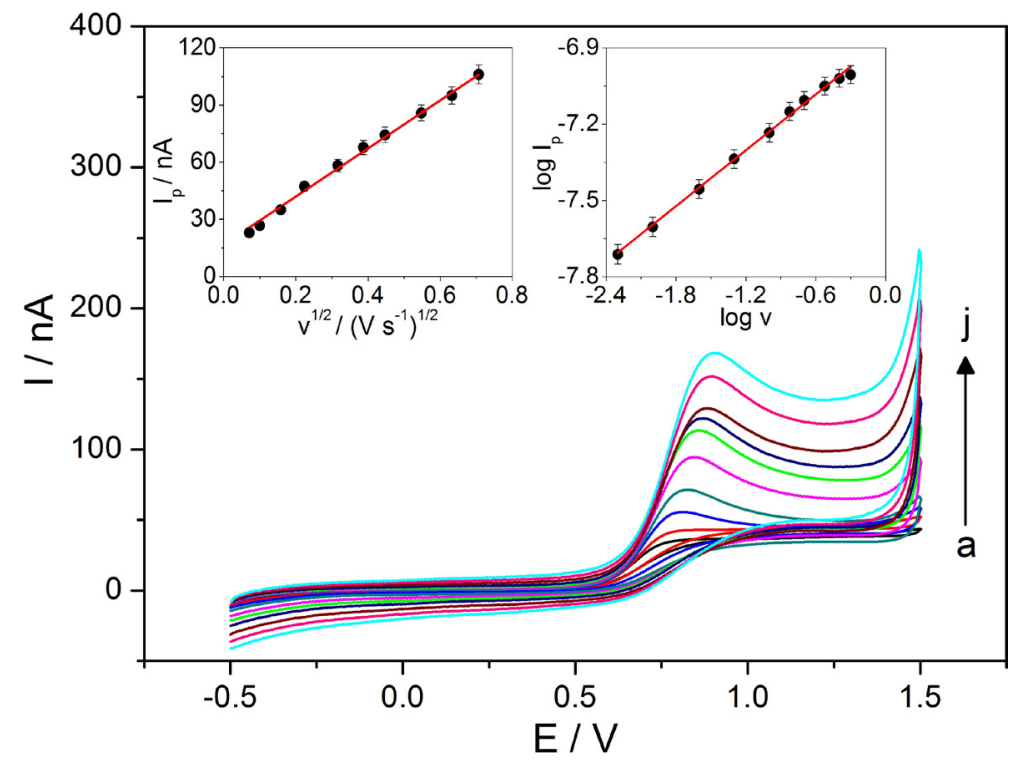

Fig. 4. $\mathrm{CV}$ records of $1.0 \times 10^{-4} \mathrm{~mol} \mathrm{~L}^{-1} \mathrm{AA}$ for different scan rates (v): (a) 0.005, (b) 0.01, (c) 0.025, (d) 0.05, (e) 0.1, (f) 0.15, (g) 0.2, (h) 0.3, (i) 0.4 and (j) $0.5 \mathrm{~V} \mathrm{~s}^{-1}$ in B-R buffer $\mathrm{pH} 4.0$ at BDD electrode. The peak current $I_{\mathrm{p}}$ as a function of square root of the scan rate $v^{1 / 2}$ and logarithmic analysis $\left(\log I_{\mathrm{p}}\right.$ as a function of $\log v$ ) are appended in the inset.

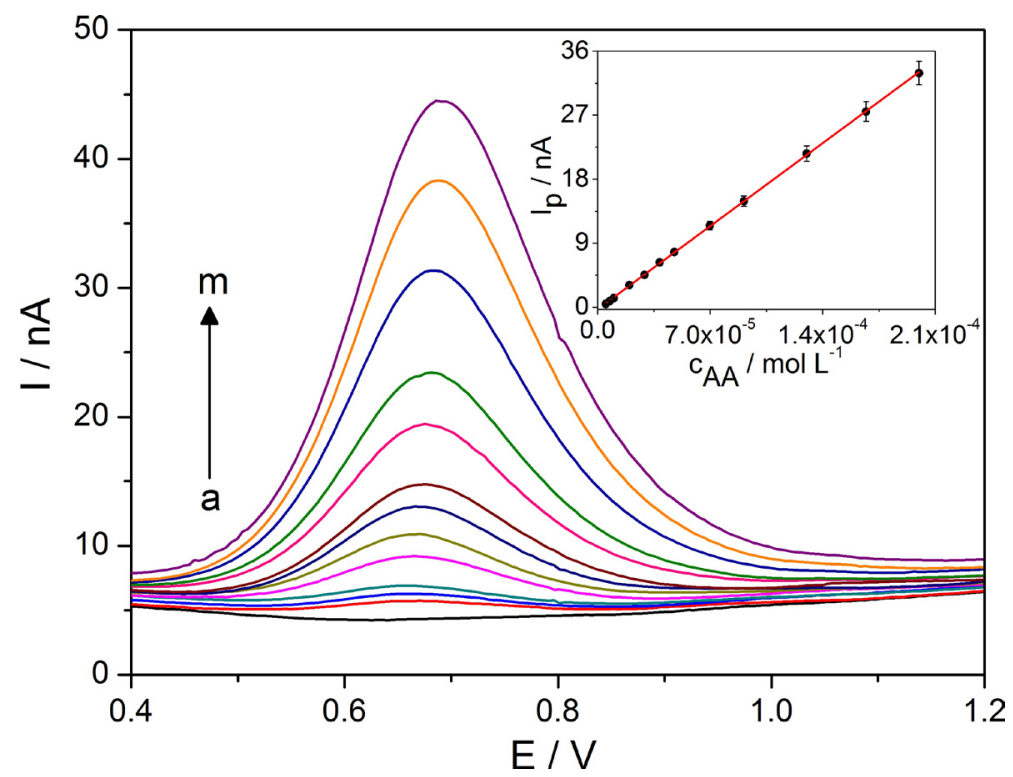

Fig. 5. DPV records for various AA concentrations: (a) 0 ; (b) $5 \times 10^{-6}$; (c) $7.4 \times 10^{-6}$; (d) $9.9 \times 10^{-6}$; (e) $2.0 \times 10^{-5}$; (f) $2.9 \times 10^{-5}$; (g) $3.9 \times 10^{-5}$; (h) $4.8 \times 10^{-5}$; (i) $7.0 \times 10^{-5}$; (j) $9.1 \times 10^{-5}$; (k) $1.3 \times 10^{-4}$; (l) $1.7 \times 10^{-4}$ and $(\mathrm{m}) 2.0 \times 10^{-4} \mathrm{~mol} \mathrm{~L}^{-1}$ in B-R buffer $\mathrm{pH} 4.0$ at BDD electrode.

DPV parameters: modulation amplitude of $50 \mathrm{mV}$, modulation time of $50 \mathrm{~ms}$ and scan rate of $10 \mathrm{mV} \mathrm{s}^{-1}$. The dependence between $I_{\mathrm{p}}$ and $c_{\mathrm{AA}}$ is appended in the inset.

between the particular peak currents $\left(I_{\mathrm{p}}\right)$ and AA concentration $\left(c_{\mathrm{AA}}\right)$ are depicted in Fig. 5. The calibration curve was obtained in triplicate for AA concentrations ranging from $5 \times 10^{-6}$ to $2 \times 10^{-4} \mathrm{~mol}$ $\mathrm{L}^{-1}$. The corresponding analytical parameters are summarized in Table 1 . The LOD and LOQ were found to be $1.1 \times 10^{-6}$ and $3.5 \times 10^{-6} \mathrm{~mol} \mathrm{~L}^{-1}$, respectively. The repeatability study was performed by measuring six successive DPV responses for $7.0 \times 10^{-5} \mathrm{~mol} \mathrm{~L}^{-1} \mathrm{AA}$ with the relative standard deviation (RSD) value of $2.3 \%$ thus proving the distinguished current response stability of BDD electrode. The accomplished analytical performance indicated the successful employement of the developed electroanalytical protocol for subsequent analytical purposes. 
Tab. 1. Analytical parameters for AA determination by DPV in B-R buffer pH 4.0 at BDD electrode $(n=3)$.

\begin{tabular}{lc}
\hline Analytical parameter & Value \\
\hline Intercept $(\mathrm{nA})$ & $(-0.25 \pm 0.06)$ \\
Slope $\left(\mathrm{nA} \mathrm{L} \mathrm{mol}{ }^{-1}\right)$ & $(1.67 \pm 0.05) \times 10^{5}$ \\
Linear concentration range $\left(\mathrm{mol} \mathrm{L}^{-1}\right)$ & $(5-200) \times 10^{-6}$ \\
Coefficient of determination & 0.999 \\
Limit of detection $(\mathrm{mol} \mathrm{L}-1)$ & $1.1 \times 10^{-6}$ \\
Limit of quantification $\left(\mathrm{mol} \mathrm{L}^{-1}\right)$ & $3.5 \times 10^{-6}$ \\
Repeatability $(\%)^{*}$ & 2.3 \\
\hline
\end{tabular}

${ }^{*}$ RSD calculated for 6 replicate DPV measurements using $7.0 \times 10^{-5} \mathrm{~mol} \mathrm{~L}^{-1} \mathrm{AA}$

The practical feasibility of the proposed protocol was undertaken by assessing the AA content in commercial pharmaceutical tablets by means of the standard addition method. An aliquot of the sample stock solution was added into the electrochemical cell with the B-R buffer $\mathrm{pH} 4.0$ followed by additions of AA standard solution. The respective DPV records with the distinct oxidation peaks of AA at $+0.7 \mathrm{~V}$ are displayed in Fig. 6 .

According to the drug leaflet of the manufacturer, the declared amount of AA was $250 \mathrm{mg}$ per tablet. The mass value reached by the proposed electroanalytical protocol using BDD electrode was found to be (304 \pm 16$)$ mg per tablet thus providing the recovery of $122 \%$. The higher recovery value reflects the fact that there is mild matrix effect present in the analyzed pharmaceutical sample. In spite of this fact, the developed method using BDD electrode could be considered as promising for the quantification of AA in pharmaceutical samples. However, to improve the analytical performance of the developed protocol (higher sensitivity and selectivity, lower LOD) and to achieve accurate results, the analysis of various pharmaceutical samples with the AA content using the particular BDD electrode should be carried out. In this respect, an optimization of DPV operation parameters such as modulation amplitude and modulation time is often preferred to be performed in the development of electroanalytical methodology. An effect of some interfering agents (usually present in pharmaceuticals such as starch, glucose, lactose, stearate, color additives) on the AA determination should be provided as well.

A comparison of the selected characteristics of the developed protocol using bare BDD electrode and several so far published electroanalytical methods for the AA determination is given in Table 2.

From these data, it can be emphasized that the interests of electroanalytical chemists have been mostly focused on nano-scaled materials and/or polymers as modifiers in CMEs for quantifying AA. Nevertheless, the LOD accomplished by applying BDD electrode in the proposed paper is several times lower than those obtained by $\mathrm{PVP} / \mathrm{Mo}(\mathrm{CN})_{8}{ }^{4-} / \mathrm{GCE}$ (Zheng et al., 2015), SWCNT/GCE (Habibi et al., 2011) and comparable with CoPc-MWCNT/GCE (Zuo et al., 2012) and IL-SWCNT/GCE (Ping et al., 2012). Owing to the above facts, it is worth men-

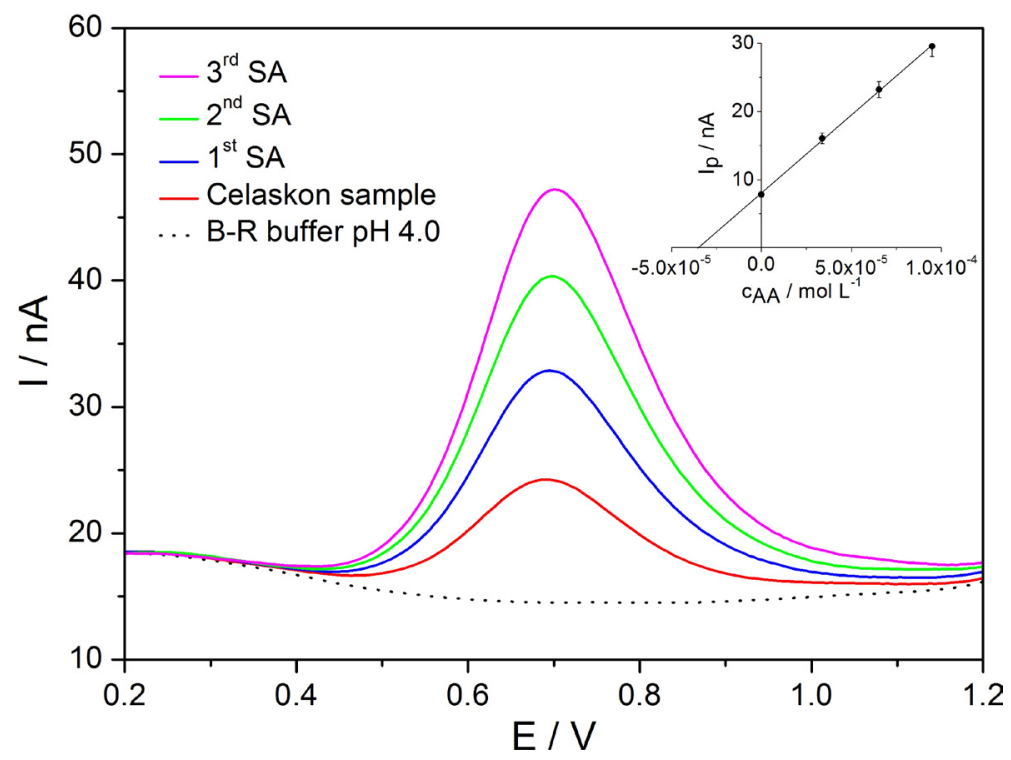

Fig. 6. DPV records for the analysis of Celaskon ${ }^{\circledR}$ with the declared content of $250 \mathrm{mg}$ AA using standard addition (SA) method in B-R buffer $\mathrm{pH} 4.0$ at BDD electrode. DPV parameters: modulation amplitude of $50 \mathrm{mV}$, modulation time of $50 \mathrm{~ms}$ and scan rate of $10 \mathrm{mV} \mathrm{s}^{-1}$. The analysis by standard addition method is depicted in the inset. 
Tab. 2. Comparison of basic characteristics of the developed protocol with the previously reported methods for the AA determination.

\begin{tabular}{|c|c|c|c|c|}
\hline Working electrode & $\begin{array}{l}\text { Supporting } \\
\text { electrolyte }\end{array}$ & $\begin{array}{c}\text { LCR } \\
\left(10^{6} \mathrm{~mol} \mathrm{~L}^{-1}\right)\end{array}$ & $\begin{array}{c}\text { LOD } \\
\left(10^{6} \mathrm{~mol} \mathrm{~L}^{-1}\right)\end{array}$ & References \\
\hline CoPc-MWCNT/GCE & PB pH 7.0 & $10-260$ & 1 & Zuo et al., 2012 \\
\hline PL-LEU/DNA/GCE & $\mathrm{PB} \mathrm{pH} 4.5$ & $5-750$ & 2 & Zheng et al., 2015 \\
\hline $\mathrm{PVP} / \mathrm{Mo}(\mathrm{CN})_{8}^{4-} / \mathrm{GCE}$ & $0.1 \mathrm{~mol} \mathrm{~L}^{-1} \mathrm{H}_{2} \mathrm{SO}_{4}$ & $980-1000$ & 5.5 & Thangamuthu et al., 2007 \\
\hline poly-Xa/MWCNT/GCE & PB pH 7.0 & $25-333$ & 0.2 & Silva et al., 2012 \\
\hline IL-SWCNT/GCE & PB pH 7.0 & $3-420$ & 1 & Ping et al., 2012 \\
\hline $\mathrm{PdNi} / \mathrm{GCE}$ & PB pH 7.4 & $10-180$ & 0.5 & Zhang et al., 2013 \\
\hline $\mathrm{Co} / \mathrm{GCE}$ & PB pH 8.0 & $0.3-100$ & 0.2 & Tian et al., 2010 \\
\hline SWCNT/GCE & PB pH 7.0 & $5-700$ & 3 & Habibi et al., 2011 \\
\hline bare BDDE & B-R pH 4.0 & $5-200$ & 1.1 & This work \\
\hline
\end{tabular}

Abbreviations: BDDE - boron-doped diamond electrode, B-R - Britton-Robinson buffer, CoPc - cobalt(II)phthalocyanine, DNA - deoxyribonucleic acid, GCE - glassy carbon electrode, IL - ionic liquid, LCR - linear concentration range, LOD - limit of detection, MWCNT - multi-walled carbon nanotube, PB - phosphate buffer, PL-LEU - poly(l-leucine), poly-Xa - poly-xanthurenic acid, PVP - poly(4-vinylpyridine), SWCNT - single-walled carbon nanotube.

tioning that the application of CMEs could also be accompanied by extending the total procedure time because of a requirement of the surface modification procedure. This step, on the one hand, enhances the sensitivity and selectivity, but on the other hand oftentimes gives the low reproducibility due to the singularity in the actual status of the CME surfaces which may constrain their reliable usage in routine analysis. Accordingly, herein presented electroanalytical protocol using BDD electrode combined with the DPV technique could meet all required characteristics for the modern electrochemical sensors (rapidity, simplicity, sufficient sensitivity, good repeatability and low cost). Overall, it may be used as modification-free electrochemical platform for determination of vitamins in pharmaceutical samples.

\section{Conclusions}

Current analytical methodologies and procedures, mostly using separation methods for the determination of AA, are relatively of high cost with time consuming analysis. In this work, the BDD electrode was executed in connection with CV and DPV techniques to study the electrochemical behavior of AA and to elaborate the advanced and simple electroanalytical protocol for its determination in pharmaceutical tablets. CV measurements manifested one irreversible and diffusion-controlled oxidation peak of
AA at $+0.87 \mathrm{~V}$. The analytical parameters using DPV were assessed as follows: $\mathrm{LOD}=1.1 \times 10^{-6} \mathrm{~mol} \mathrm{~L}^{-1}$, $\mathrm{LOQ}=3.5 \times 10^{-6} \mathrm{~mol} \mathrm{~L}^{-1}$, the linear concentration range of $(5-200) \times 10^{-6} \mathrm{~mol} \mathrm{~L}^{-1}\left(R^{2}=0.999\right)$ and the repeatability with $\mathrm{RSD}=2.3 \%$. The developed procedure was applied to the analysis of the Celaskon ${ }^{\circledR}$ 250 pharmaceuticals without a complex sample pretreatment with the recovery of $122 \%$. To sum up, the innovative aspect of this electroanalytical protocol lies in the application of BDD electrode as advanced and modification-free electrode platform which may replace the conventional carbonaceous electrode materials (graphite, carbon paste, glassy carbon and gold) in drug analysis.

\section{Acknowledgement}

This work has been supported by the Grant Agency of the Slovak Republic (grant No. 1/0489/16 and 1/0558/17) and Grant scheme for Support of Young Researchers and Excellent Teams of Young Researchers.

\section{References}

Bi H, Fernandes AC, Cardoso S, Freitas P (2016) Sens. Actuators B 224: 668-675.

Cinková K, Švorc L', Šatkovská P, Vojs M, Michniak P, Marton M (2016) Anal. Lett. 49: 107-121.

Compton RG, Banks CE, Undestanding Voltammetry, 2nd Edition, Imperial College Press, London, 2010.

Ensafi AA, Taei M, Khayamian T, Arabzadeh A (2010) Sens. Actuators B 147: 213-221. 
Falkova MT, Bulatov AV, Pushina MO, Ekimov AA, Alekseeva GM, Moskvin LN (2015) Talanta 133: 82-87.

Fong JFY, Chin SF, Ng SM (2016) Biosens. Bioelectron. 85: 844-852.

Guanghan L, Yu W, Leiming Y, Shuanglong H (1994) Food Chem. 51: 237-239.

Habibi B, Jahanbakhshi M, Pournaghi-Azar MH (2011) Anal. Biochem. 411: 167-175.

Lee C, Seo JY, Jin EH, Lim JH, Kang HY, Yang JI, Chung SJ, Yang SY, Kim JS (2016) Gastrointest. Endosc. 83: AB226.

Mason SA, Della Gatta PA, Snow RJ, Russell AP, Wadley GD (2016) Free Radical Biol. Med. 93: 227-238.

Muñoz RAA, Matos RC, Angnes L (2001) Talanta 55: $855-860$

Ping J, Wang Y, Wu J, Ying Y, Ji F (2012) Food Chem. 135: 362-367.

Pisoschi AM, Pop A, Negulescu GP, Pisoschi A (2011) Molecules 16: 1349-1365.
Silva FAS, Lopes CB, Kubota LT, Lima PR, Goulart MOF (2012) Sens. Actuators B 168: 289-296.

Svítková J, Ignat T, Švorc L, Labuda J, Barek J (2016) Crit. Rev. Anal. Chem. 46: 248-256.

Švorc L, Cinková K, Samphao A, Stanković DM, Mehmeti E, Kalcher K (2015) J. Electroanal. Chem. 744: 37-44.

Thangamuthu R, Kumar SMS, Pillai KC (2007) Sens. Actuators B 120: 745-753.

Tian K, Bian J, Wang B, Qi Y (2010) Electrochim. Acta 55: 3083-3088.

Zhang X, Cao Y, Yu S, Yang F, Xi P (2013) Biosens. Bioelectron. 44: 183-190.

Zheng X, Guo Y, Zheng J, Zhou X, Li Q, Lin R (2015) Sens. Actuators B 213: 188-194.

Zuo X, Zhang H, Li N (2012) Sens. Actuators B 161: 1074-1079.

Zuo R, Zhou S, Zuo Y, Deng Y (2015) Food Chem. 182: 242-245. 